\title{
Alternativas durante el covid-19 para profesorado universitario dedicado a la enseñanza de la educación física*
}

\author{
Javier Arturo Hall-López ${ }^{* *}$ \\ Paulina Yesica Ochoa-Martínez ${ }^{* * *}$ \\ Edgar Ismael Alarcón Meza****
}

Recibido: 17 de noviembre de 2020

Aceptado: 3 de diciembre de 2020

Citar como:

Hall-López, J. A., Ochoa-Martínez, P. Y. y Alarcón Meza, E. I. (2021). Alternativas durante el covid 19, para profesorado universitario dedicado a la enseñanza de la educación física. Revista de Investigación Cuerpo, Cultura y Movimiento, 11(1). https://doi.org/10.15332/2422474X.6464

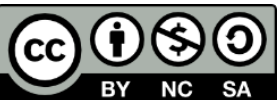

\footnotetext{
* Artículo de reflexión. Financiado por la Universidad Autónoma de Baja California (UABC), México proyecto clave 149/2604. Vinculación con el Cuerpo Académico Ciencias de la Actividad Física y Deporte UABC-175 de la Facultad de Deportes (UABC). Mexicali, Baja California. México.

** Doctor en Enseñanza de la Actividad Física y Deporte por la Universidad de Huelva, y Doctor en Medicina del Deporte por la Universidad Católica Nuestra Señora de la Asunción. Profesor-investigador de tiempo completo de la Universidad Autónoma de Baja California, Facultad de Deportes, México. Correo electrónico: javierhall@uabc.edu.mx; ORCID: https://orcid.org/0000-0002-7808-0181

${ }_{* * *}$ Doctora en Enseñanza de la Actividad Física y Deporte por la Universidad de Huelva, y Doctora en Medicina del Deporte por la Universidad Católica Nuestra Señora de la Asunción. Profesora-investigadora de tiempo completo de la Universidad Autónoma de Baja California, Facultad de Deportes, México. ORCID: https://orcid.org/0000-0001-8107-4906

${ }_{* * * *}$ Dr. Universidad Autónoma de Baja California, Facultad de Deportes, México Correo electrónico eiam@uabc.edu.mx; ORCID: https://orcid.org/0000-0003-1469-4712
} 


\section{Resumen}

En este artículo se analizaron investigaciones originales sobre el covid-19 y la educación física para hallar campos de acción y exponer puntos clave que puede considerar el profesorado universitario dedicado a la enseñanza de la actividad física. El profesorado se ha vinculado para realizar prácticas docentes en la educación básica en México, considerando alternativas como la adecuación curricular, la crisis alterna de sedentarismo y obesidad en México con la pandemia por covid-19, y la actividad física con intensidad moderada a vigorosa como parte esencial y permanente de la clase de educación física.

Palabras clave: actividad física, coronavirus, universidad.

\section{Alternatives during COVID-19 for university professors dedicated to teaching physical education}

\section{Abstract}

In this article, original research on COVID-19 and physical education were analyzed to find fields of action and present key aspects to be considered by university teachers dedicated to teaching physical activity. The faculty has engaged to carry out teaching practices in basic education in Mexico, considering alternatives such as curricular adaptation, the alternative crisis of sedentary lifestyle and obesity in Mexico with the COVID-19 pandemic, and physical activity with moderate to vigorous intensity as an essential and permanent part of the physical education class.

Keywords: physical activity, coronavirus, university.

\section{Introducción}

El aislamiento social por covid-19 se llevó a cabo como una adaptación y colaboración de todos los sectores sociales para prevenir los contagios y el riesgo de muerte en la población (Ramos, 2020). El aislamiento social 
incluyó la suspensión súbita de múltiples actividades cotidianas, incluyendo la educación universitaria, donde las acciones académicas transitaron de una educación presencial a una educación no presencial (Wang et ál.,2020). Simultáneamente al aislamiento social, el profesorado universitario se capacitó y obtuvo experiencia en el uso de tecnologías y la enseñanza virtual a los estudiantes universitarios (Lloyd, 2020), ajustando el proceso cotidiano de enseñanza-aprendizaje, para innovar y ejecutar acciones pedagógicas mediante la educación a distancia (García-Peñalvo et ál.,2020). En esta circunstancia, e inesperadamente, las universidades en sus programas educativos adecuaron las competencias que deberían obtener en las unidades de aprendizaje con el sector público y privado (Emiliozzi et ál., 2011). Por otro lado, a pesar de la responsabilidad social en las funciones sustantivas de las universidades, derivado de la emergencia sanitaria, se suspendieron las actividades previamente planeadas de servicio o prácticas profesionales (Martínez-Pichardo y Hernández-Oliva, 2013), repercutiendo negativamente en la vinculación de las universidades públicas para iniciar labores académicas y de investigación (Alcántar Enríquez y Arcos Vega, 2009).

Para el caso de los programas educativos de grado y posgrado dedicados a la enseñanza de la educación física, educación deportiva o ciencias del ejercicio se tenía contemplado obtener competencias vinculadas a la educación básica y formar alianzas como se establece en la guía para responsables políticos en educación física de la Organización de las Naciones Unidas para la Educación, la Ciencia y la Cultura (Unesco). Para lograr la calidad educativa en la educación física (McLennan y Thompson 2015), a partir de lo anterior, se presenta una reflexión sobre los resultados de investigaciones sobre la crisis sanitaria por covid-19, recurriendo a fuentes y referencias sobre la actividad física y educación física en el contexto educativo, con la intención de que los licenciados en actividad 
física y deporte en formación de la Facultad de Deportes de la Universidad Autónoma de Baja California lo tengan en cuenta para realizar su labor docente durante la pandemia por covid-19 (Gómez Calvo, 2020; BaenaMorales et ál., 2020). Esto para exponer tres puntos clave como alternativas para ser considerados por el profesorado universitario dedicado a la enseñanza de la educación física, que por medio de su labor se vincula para realizar prácticas docentes en la educación básica en México.

\section{Adecuación curricular en planes de estudios sobre contenidos relacionados con el covid-19 y habilidades digitales}

La adecuación curricular representa un reto para el profesorado universitario dedicado a la enseñanza de la cultura física de universidades adscritas a la Asociación Mexicana de Instituciones Superiores de Cultura Física (AMISCF, 2012), ya que el aislamiento social para prevenir la infección por covid-19 ha limitado la práctica de actividad física, ejercicio y deporte; esta situación ha entorpecido la labor docente de actualización de los programas educativos de grado y posgrado de acuerdo con las tendencias internacionales (Blocken et ál., 2020). De igual forma, esto ha condicionado las medidas tomadas por los profesionales de la educación física para realizar su labor docente durante la crisis sanitaria (Gómez Calvo, 2020).

En concordancia con lo anterior, se presenta el ejemplo de la Universidad Autónoma de Baja California (UABC), que en su Plan de Desarrollo Institucional PDI 2019-2023 (2020), establece la cultura digital como política, estrategia y acción institucional. Esta plantea como objetivo incorporar la cultura digital en la realización de las funciones sustantivas y de gestión de la universidad con base en esquemas de colaboración y aprovechamiento de las tecnologías digitales. La unidad académica de la 
UABC, responsable de la enseñanza de la actividad física es la Facultad de Deportes UABC (2020), cuya misión es la formación de profesionales competentes en la actividad física y en el deporte para impulsar el desarrollo de la sociedad a través de la cultura física, y con una adecuación curricular en unidades de aprendizaje de sus programas educativos de Licenciatura en Actividad Física y Deporte (2012) y Maestría en Educación Física y Deporte (2020), puede adaptar sus contenidos para la enseñanza virtual de la educación física en educación básica.

En ese sentido, en México, de acuerdo al plan de estudios vigente de educación física establecido por la Secretaría de Educación Pública (2017), como área de desarrollo personal y social, se pueden asociar los ámbitos del perfil de egreso denominados habilidades digitales y atención al cuerpo y la salud; esta conexión se puede fundamentar en la necesidad social y en los contenidos curriculares de planes de estudios universitarios dedicados a la enseñanza de la educación física, como se expresa a continuación; esto teniendo como fuente el programa de aprendizajes clave para la educación integral de educación física para la educación básica en el plan y programa de estudio, orientaciones didácticas y sugerencias de evaluación:

Habilidades digitales: "Está familiarizado con el uso básico de herramientas digitales a su alcance" (SEP, 2017).

Atención al cuerpo y la salud: "Reconoce su cuerpo, resuelve retos y desafíos mediante el uso creativo de sus habilidades corporales. Toma decisiones informadas sobre su higiene y alimentación. Participa en situaciones de juego y actividad física procurando la convivencia sana y pacífica" (SEP, 2017). 


\section{Triple crisis de salud en México: covid-19, obesidad y sedentarismo}

Desafortunadamente, se estima que la emergencia sanitaria por covid-19 también aumentará de manera simultánea la crisis actual de sedentarismo en la sociedad (Hall et ál., 2020). Múltiples estudios documentan la importancia de la actividad física y el deporte como una ocupación con competencias claves para generar estrategias de salud e incrementar la actividad física en la población (McKenzie y Lounsbery, 2013; McKenzie y Lounsbery, 2014; Webster et ál., 2015). Durante el aislamiento social por covid-19 se estima que habrá un aumento de enfermedades cardiovasculares asociadas al sedentarismo (Lippi et ál., 2020), lo que es alarmante dados los resultados de la Encuesta Nacional de Salud y Nutrición (Ensanut), cuyas prevalencias combinadas de sobrepeso y obesidad están reportadas en tres de cada diez niños y niñas en edad escolar (5 a 11 años); dichos valores son de los más altos a nivel mundial (Pérez-Herrera y Cruz-López, 2019; Campos Rodríguez et ál., 2020).

Teniendo en cuenta lo establecido por Rundle et ál., (2020), se estima que después de la pandemia por covid-19 los estudiantes en edad escolar presentarán un aumento de masa corporal asociada al sedentarismo por el confinamiento y los impedimentos que este ha traído para practicar actividad física. El problema se hace más grave debido a que revisiones sistemáticas y metaanálisis asocian la presencia de obesidad y enfermedades crónicas no trasmisibles con los casos de infección severa por covid-19 en la población (Zhou et ál., 2020); en México, se presenta el mismo patrón epidemiológico (Denova-Gutiérrez et ál.,2020).

\section{Actividad física con intensidad moderada a vigorosa y alfabetización corporal}

Durante el periodo de aislamiento social por covid-19 se ha recomendado la práctica de actividad física con intensidades moderada a vigorosa en 
edades infantiles (Cossio-Bolaños, 2020). Esta también es recomendada por la Organización Mundial de la Salud (OMS, 2010), que establece que practicar actividad física con intensidad moderada a vigorosa durante 60 minutos diarios es beneficioso para la salud y es la actividad más efectiva durante el confinamiento (Dixit, 2020).

En educación física las intervenciones educativas exitosas han mejorado la motricidad de los estudiantes y disminuido la grasa corporal, utilizando actividad física con intensidad moderada a vigorosa (Hall-López et ál., 2017; Piña Díaz et ál., 2020), lo cual, acorde con el sistema educativo mexicano, es congruente con el perfil de egreso denominado atención al cuerpo y la salud, que se puede trabajar en el componente curricular de educación física como área de desarrollo personal social (SEP, 2017). Durante la emergencia sanitaria, el profesorado de educación física puede orientar contenidos digitales, para fomentar tareas motrices en educación física y guiar al estudiantado a participar en actividad física moderada a vigorosa por lo menos el $50 \%$ del tiempo de la clase. Esto con el fin de obtener una adecuada alfabetización corporal, que se alcanza cuando el estudiantado encuentra diversidad de oportunidades en cada etapa y edad (Bolaños Motta et ál., 2018). En ese sentido, se ha recomendado el uso de tecnología en la emergencia sanitaria para la clase de educación física (Hall-López y Ochoa-Martínez, 2020).

Existen múltiples intervenciones exitosas en educación física en contextos escolares que han utilizado la tecnología y ambientes virtuales para incrementar la práctica de la actividad física y mejorar los hábitos de salud del estudiantado (Bezkopylnyi et ál., 2020; Oleniev, 2020). Se ha encontrado que la actividad física antes y durante la pandemia por covid-19 ha disminuido en sujetos físicamente activos, como atletas (Alarcón Meza y Hall-López, 2020). De igual forma, ese mismo patrón se ha encontrado en el profesorado de educación física, cuyo trabajo implica 
acciones motrices moderadas para conducir la clase de educación física y que no realizó dicho gasto energético durante la pandemia (Hall-López, 2020). Además, se ha identificado que en este periodo las estudiantes tuvieron menor actividad física (Hall-López et ál., 2020); por tal razón, es fundamental abordar e intervenir este problema derivado de la crisis sanitaria desde el ámbito de la educación física, para evaluar y reducir sus efectos en la salud.

\section{Conclusión}

Las universidades formadoras en la enseñanza de educación física tienen un gran reto y una gran oportunidad para brindar una atención de calidad al vincularse con la educación básica. De esta manera, pueden estar preparadas ante posibles rebrotes de covid-19 que alarguen la pandemia y limiten la educación tradicional. Los planes de desarrollo de las universidades resaltan la cultura y habilidades digitales, que en los programas educativos de grado y posgrado son herramientas útiles para adaptarse a la práctica docente en educación física y fomentar aprendizajes esperados que promuevan la actividad física moderada a vigorosa y tiendan a la alfabetización corporal, para coadyuvar a la solución de problemas de salud derivados de la obesidad en México.

\section{Referencias}

Alarcón Meza, E. y Hall-López, J. (2020). Physical activity in university student athletes, prior and in confinement due to pandemic associated with COVID-19. Retos, (39), 572-575. https://doi.org/10.47197/retos.voi39.81293

Alcántar Enríquez, V. y Arcos Vega, J. (2009). La vinculación como factor de imagen y posicionamiento de la Universidad Autónoma de Baja California, México, en su entorno social y productivo. REDIE: Revista electrónica de investigación educativa, 11(1),1-20. https://www.redalyc.org/articulo.oa?id=15511137011. 
Asociación Mexicana de Instituciones Superiores de Cultura Física [AMISCF]. (2012).

[Página web]. Benemérita Universidad Autónoma de Puebla.

http://cmas.siu.buap.mx/portal_pprd/wb/amiscf

Baena-Morales, S., López-Morales, J. y García-Taibo, O. (2020). La intervención docente en educación física durante el periodo de cuarentena por COVID-19. Retos, (39), 388-395. https://doi.org/10.47197/retos.voi39.80089

Bezkopylnyi, O., Bazylchuk, O., Sushchenko, L., Bazylchuk, V., Dutchak, Y. y Ostapenko, H. (2020). Peculiarities of application of interactive educational technologies in training of future teachers of physical culture to work with health protection in secondary school. Journal of Physical Education and Sport, 2O(1), 291-297. https:// doi:10.7752/jpes.2020.slo40

Blocken, B., Malizia, F., van Druenen., T. y Marchal, T. (2020). Towards aerodynamically equivalent COVID-19 1.5 m social distancing for walking and running. Urban Physics, Wind Engineering \& Sports Aerodynamics.

https://www.euroga.org/system/1/user_files/files/ooo/045/111/45111/150d3060c Loriginal/Social Distancing v20 White Paper.pdf

Bolaños Motta, J., Pérez Rodríguez, M. y Casallas Forero, E. (2019). Alfabetización Corporal. Una propuesta de aula desde la psicomotricidad. Estudios Pedagógicos, 44(3), 23-34. https://doi:10.4067/So718-07052018000300023

Campos Rodríguez, A., Romero García, J., Hall-López, J. y Ochoa Martínez, P. (2020). Overview of overweight and obesity in Latin American schools. Retos, o(39). https://recyt.fecyt.es/index.php/retos/article/view/78426/50420

Chen, P., Mao, L., Nassis, G. P., Harmer, P., Ainsworth, B. E. y Li, F. (2020). Coronavirus disease (COVID-19): The need to maintain regular physical activity while taking precautions. Journal of sport and health science, 9(2), 103-104. https://doi.org/10.1016/j.jshs.2020.02.001

Cossio-Bolaños, M. (2020). Actividad física en tiempos de cuarentena por el COVID-19 en niños y adolescentes. Revista peruana de ciencias de la actividad física y del deporte, 7(2), 1-2. https://www.rpcafd.com/index.php/rpcafd/article/view/92

Revista de Investigación Cuerpo, Cultura y Movimiento

ISSN: 2248-4418 | e-ISSN: 2422-474X | DOI: https://doi.org/10.15332/2422474X

Vol. 11 N.o 1 | enero-junio de 2021 
Denova-Gutiérrez, E., Lopez-Gatell, H., Alomia-Zegarra, J. L., López-Ridaura, R., Zaragoza-Jimenez, C. A., Dyer-Leal, D. D., Cortés-Alcala, R., Villa-Reyes, T., Gutiérrez-Vargas, R., Rodríguez-González, K., Escondrillas-Maya, C., BarrientosGutiérrez, T., Rivera, J. A. y Barquera, S. (2020). The Association of Obesity, Type 2 Diabetes, and Hypertension with Severe Coronavirus Disease 2019 on Admission Among Mexican Patients. Obesity (Silver Spring, Md.), o, 1-7. https://doi.org/10.1002/oby.22946

Dixit, S. (2020). Can moderate intensity aerobic exercise be an effective and valuable therapy in preventing and controlling the pandemic of COVID-19? Medical Hypotheses. https://doi.org/10.1016/j.mehy.2020.109854

Emiliozzi, S., Vasen, F. y Palumbo, M. M. (2011). Desafíos para la vinculación entre la universidad pública y demandas de actores sociales y gubernamentales. Espacio Abierto, 2o(2), 329-341. https://www.redalyc.org/articulo.oa?id=12218869006

García-Peñalvo, F. J., Corell, A., Abella-García, V. y Grande, M. (2020). La evaluación online en la educación superior en tiempos de la COVID-19. Education in the Knowledge Society, 21, 12. https://doi.org/10.14201/eks.23013

Gómez Calvo, J. L. (2020). Y de pronto el covid-19 nos hizo despertar. Revista Española de Educación Física y Deportes, 429, 95-100. https://www.reefd.es/index.php/reefd/article/view/903/749

Hall-Lopez, J. A. (2020). Physical activity levels in physical education teachers before and during school suspension brought by the COVID-19 quarantine. Facta Universitatis, Series Physical Education and Sport, 19(2) 179-188. https://doi.org/10.22190/FUPES200607045H

Hall-López, J. A., Ochoa-Martínez, P. Y. y Alarcón Meza, E. I. (2020). Actividad física, acorde a sexo, en estudiantes de secundaria antes y durante del distanciamiento social por el COVID-19. Revista Espacios, 41(42) 93-99. https://www.revistaespacios.com/a20v41n42/20414208.html

Hall, G., Laddu, D. R., Phillips, S. A., Lavie, C. J., y Arena, R. (2020). A tale of two pandemics: How will COVID-19 and global trends in physical inactivity and sedentary behavior affect one another? Progress in cardiovascular diseases, Soo33-062O(20)30077-3. https://doi.org/10.1016/j.pcad.2020.04.005 
Hall-López, J. A. y Ochoa-Martínez, P. Y. (2020). Enseñanza virtual en educación física en primaria en México y la pandemia por COVID-19. Ciencias de la Actividad Física UCM, 21(2), 1-7. https://doi.org/10.29035/rcaf.21.2.4

Hall-López, J. A., Ochoa-Martínez, P. Y., Zamudio Bernal, A., Sánchez León, R., Uriarte Garza, L., Almagro, B., Moncada-Jiménez, J. y Sáenz-López Buñuel, P. (2017). Efecto de un programa de actividad física de moderada a vigorosa de diez meses sobre el vo2máx y el porcentaje de grasa corporal en niños con sobrepeso y obesidad. MHSalud: Revista en Ciencias del Movimiento Humano y Salud, 14(1), 1-14. http://dx.doi.org/10.15359/mhs.14-1.6

Licenciatura en Actividad Física y Deporte Universidad Autónoma de Baja California LAFD UABC (2012) (UABC). Plan de estudio 2012-2 unidades de aprendizaje Educación Ambiental y Deporte. http://deportes.uabc.mx/images/UABC/plan2012/OBasicas/PUA-EducacionAmbiental-y-Deporte.pdf

Lippi, G., Henry, B. M. y Sanchis-Gomar, F. (2020). Physical inactivity and cardiovascular disease at the time of coronavirus disease 2019 (COVID-19). European Journal of Preventive Cardiology, o(o) 1-3. https://doi.org/10.1177/2047487320916823

Lloyd, M. (2020). Desigualdades educativas y la brecha digital en tiempos de COVID-19. En H. Casanova Cardiel (Coord.), Educación y pandemia: una visión académica (pp. 115-121). Universidad Nacional Autónoma de México, Instituto de Investigaciones sobre la Universidad y la Educación. http://132.248.192.241:8080/xmlui/bitstream/handle/IISUE UNAM/546/Lloyd M 2020 Desigualdades educativas.pdf?sequence $=1$ \&isAllowed $=y$

Maestría en Educación Física y Deporte Escolar [MEFDE], y Universidad Autónoma de Baja California [UABC]. (2020). Evaluación del Aprendizaje en la Educación Física y Diseño de Currículo en Educación Física y Deporte Escolar [Página web]. Maestría en Educación Física y Deporte Escolar. http://deportes.uabc.mx/posgrado/index.php/plan-de-estudio/plan-2020-1

McKenzie, T. L. y Lounsbery, M. A. (2014). The pill not Taken: Revisiting Physical Education Teacher Effectiveness in a Public Health Context. Research quarterly for exercise and sport, 85(3), 287-292. https://doi.org/10.1080/02701367.2014.931203 
McKenzie, T. L. y Lounsbery, M. A. (2013). Physical education teacher effectiveness in a public health context. Research quarterly for exercise and sport, 84(4), 419-430. https://doi.org/10.1080/02701367.2013.844025

Martínez-Pichardo, P. J. y Hernández-Oliva, A. V. (2013). Responsabilidad social universitaria: un desafío de la universidad pública mexicana. Contribuciones desde Coatepec, (24), 85-103. https://www.redalyc.org/pdf/281/28126456001.pdf

McLennan, N. y Thompson, J. (2015). Educación física de calidad (EFC): guía para los responsables políticos. Organización de las Naciones Unidas para la Educación, la Ciencia y la Cultura Unesco. https://unesdoc.unesco.org/ark:/48223/pfooo0231340

Facultad de Deportes [FD] y Universidad Autónoma de Baja California [UABC]. (2020). Misión de la Facultad de Deportes [Página web]. Facultad de Deportes de la Universidad Autónoma de Baja California. http://deportes.uabc.mx/index.php/sobre-nosotros/mision-vision

Oleniev, D. (2020). Research of health-preserving technologies in the system of physical education of students. Scientific Journal of National Pedagogical Dragomanov University, 2(122), 130-134. https://doi.org/10.31392/NPUnc.series15.2020.2(122).26

Organización Mundial de la Salud (2010). Recomendaciones mundiales sobre actividad fisica para la salud.

https://apps.who.int/iris/bitstream/handle/10665/44441/9789243599977 spa.p df

Pérez-Herrera, A. y Cruz-López, M. (2019). Situación actual de la obesidad infantil en México. Nutrición Hospitalaria, 36(2), 463-469.

https://dx.doi.org/10.20960/nh.2116

Piña Díaz, D., Ochoa-Martínez, P. Y., Hall-López, J. A., Reyes Castro, Z., Alarcón Meza, E., Monreal Ortiz, L. y Sáenz-López Buñuel, P. (2020). Efecto de un programa de educación física con intensidad moderada vigorosa sobre el desarrollo motor en niños de preescolar. Retos, 38(38), 363-368.

https://recyt.fecyt.es/index.php/retos/article/view/73818

Plan de Desarrollo Institucional de la Universidad Autónoma de Baja California PDI 2019-2023. (2020). Programa institucional, cultura digital.

http://www.uabc.mx/planeacion/pdi/2015-2019/

Revista de Investigación Cuerpo, Cultura y Movimiento

ISSN: 2248-4418 | e-ISSN: 2422-474X | DOI: https://doi.org/10.15332/2422474X

Vol. 11 N.o 1 | enero-junio de 2021 
Ramos, C. (2020). Covid-19: la nueva enfermedad causada por un coronavirus. Salud Pública de México, 62(2), 225-227. http://dx.doi.org/10.21149/11276

Rundle, A. G., Park, Y., Herbstman, J., Kinsey, E. W. y Wang, Y. C. (2020). COVID-19Related School Closings and Risk of Weight Gain Among Children. Obesity (Silver Spring), 28(6), 1008-1009. https://doi.org/10.1002/oby.22813

Secretaria de Educación Pública. (2017). Aprendizajes Clave para la Educación Integral. Educación Física. Educación Básica. Plan y Programas de Estudio y sugerencias de evaluación. Primera edición. ISBN: 978-607-97644-4-9. https://www.aprendizajesclave.sep.gob.mx/

Wang, C., Cheng, Z, Yue, X. G., McAleer y M. Risk (2020). Management of COVID-19 by Universities in China. Journal of Risk and Financial Management, 13(2), 1-6. https://doi.org/10.3390/jrfm13020036

Webster, C. A., Webster, L., Russ, L., Molina, S., Lee, H. y Cribbs, J. (2015). A systematic review of public health-aligned recommendations for preparing physical education teacher candidates. Research quarterly for exercise and sport, 86(1), 30-39. https://doi.org/10.1080/02701367.2014.980939

Zhou, Y., Yang, Q., Chi, J., Dong, B., Lv, W., Shen, L. y Wang, Y. (2020). Comorbidities and the risk of severe or fatal outcomes associated with coronavirus disease 2019: A systematic review and meta-analysis. International journal of infectious diseases: IJID: official publication of the International Society for Infectious Diseases, 99, 47-56. https://doi.org/10.1016/j.ijid.2020.07.029

Revista de Investigación Cuerpo, Cultura y Movimiento ISSN: 2248-4418 | e-ISSN: 2422-474X | DOI: https://doi.org/10.15332/2422474X Vol. 11 N.o 1 | enero-junio de 2021 